\title{
Diagnostic and prognostic value of circulating miR-221 for extranodal natural killer/T-cell lymphoma
}

\author{
Hong-Qiang Guo ${ }^{\mathrm{a}, \mathrm{b}, \mathrm{c}, 1}$, Guo-Liang Huang ${ }^{\mathrm{b}, \mathrm{d}, 1}$, Cheng-Cheng Guo ${ }^{\mathrm{a}, \mathrm{b}, 1}$, Xing-Xiang $\mathrm{Pu}^{\mathrm{a}, \mathrm{b}}$ and \\ Tong-Yu Lin ${ }^{\mathrm{a}, \mathrm{b}, *}$ \\ ${ }^{a}$ Chemotherapy Department, Cancer Center, the University of Sun Yat-Sen, Guangzhou, Guangdong, China \\ ${ }^{\mathrm{b}}$ State Key Laboratory of Oncology in South China, Guangzhou, Guangdong, China \\ ${ }^{\mathrm{c}}$ The Affiliated Cancer Hospital of Zhengzhou University, Zhengzhou, Henan, China \\ ${ }^{\mathrm{d}}$ Cancer Research Center, Guangdong Medical College, Dongguan, China
}

\begin{abstract}
Extra-nodal natural killer T-Cell (NK/T-cell) lymphoma is a progressive cancer with poor prognosis due to the lack of disease specific treatment. To develop specific therapeutic strategies, it is essential to identify tumor markers. Recent studies show that circulating microRNA (miRNA) may serve as diagnostic and/or prognostic markers for some diseases. To explore miRNAs as potential diagnostic and/or prognostic markers of NK/T-cell lymphoma, in our study, we compared circulating miR-221 levels in 79 patients and 37 normal subjects by real-time PCR amplification directly from plasma samples, and correlated patient's miR-221 levels with their clinic features and treatment outcomes. We observed a significant difference between the patient and control groups ( $p=0.038$ ), and a correlation of plasma miR-221 level in patient with sex, as well as a reverse correlation with performance status and the overall survival after treatment. Univariate and multivariate analyses further revealed that plasma miR-221 level, age, B symptoms, LDH level and complete response after primary treatment all present prognostic values when judged by overall survival (OS). Together, our results show that it is feasible to perform direct amplification of plasma miRNAs without total RNA extraction, and plasma miR-221 may be a diagnostic and prognostic marker for NK/T-cell lymphoma.
\end{abstract}

Keywords: microRNA, NK/T-cell lymphoma, prognosis, quantitative real-time PCR

\section{Introduction}

Extra-nodal natural killer T-Cell (NK/T-cell) lymphoma is a distinct entity of malignant lymphomas in the WHO classification system and a disease more frequently occurring in Asia and Latin America than in Europe and North America. While it comprises only $0.44 \%$ of all extranodal lymphomas and $0.17 \%$ of all malignant lymphomas in Western countries, it accounts approximately $2.6 \%-10.7 \%$ of non-Hodgkin's

\footnotetext{
${ }^{1}$ These authors contributed equally to this study.

* Corresponding author: Tong-Yu Lin, 651 Dongfeng East Road, Guangzhou, Guangdong 510060, China. Tel.: +86 020 87343351; E-mail: tongyulin@hotmail.com.
}

lymphomas and 40\%-74\% of all nasal and nasopharyngeal lymphomas in Asia [1-4]. NK/T-cell lymphoma is a very aggressive disease with poor prognosis. It is often treated similarly as for other progressive nonHodgkin's lymphomas, but the tumor is somehow resistant to chemotherapy, especially to regimens containing anthracyclines such as the most commonly used CHOP regimens. Therefore, to develop treatment specifically targeting this tumor, it is essential to identify effective diagnostic and prognostic markers.

As a growing class of small ( $\sim 22 \mathrm{bp})$ endogenous non-coding RNAs, microRNAs (miRNAs) modulate gene expression mainly by base-pairing to the 3/-UTR of the target mRNA, which in turn leads to translational repression, mRNA cleavage, or destabilization [5, 
6]. Since the discovery of the first miRNA lin-4 in 1993 [7,8], hundreds of miRNAs have been identified in plants, animals, and viruses by molecular cloning and bioinformatic approaches. The miRNA-mediated posttranscriptional gene regulation has been increasingly recognized as an important mechanism of regulation on many cellular processes, both physiologic and pathologic [9-11].

Several miRNA species have also been shown to play important roles in drug resistance in cancer cells. For example, Cui et al. reported that levels of miR-221 and miR-222 are significantly elevated in MCF-7/ADR cells that are resistant to adriamycin compared with its parental MCF-7 cells [12]. Olga Kovalchuk reported that microRNA-451 regulates the expression of multidrug resistance gene, and transfection of the MCF7/DOX-resistant cells with microRNA-451 resulted in the increased sensitivity of cells to adriamycin [13].

Because NK/T-cell lymphoma is often treated with adriamycin-containing regimen, to investigate whether plasma miRNAs such as miR-221 is correlated with the disease status and its treatment outcomes, we compared circulating miR-221 levels in NK/T-cell lymphoma patients and healthy subjects, and analyzed the patient group for the correlation of plasma miR-221 levels with the clinic features and long term disease outcomes after treatment with adriamycin-containing regimens.

\section{Materials and methods}

\subsection{Study subjects and diagnosis}

The study protocol was approved by Institutional Review Board (IRB). Seventy nine patients with NK/Tcell lymphoma receiving treatment in our hospital in 2004-2008 and 37 healthy individuals were enrolled in the study. All study subjects provided their written informed consent. The median age of patients was 41 years (ranged 16-74 years), and the male to female ratio was 2.16:1. The age range of 37 controls was from 23 to 64 year old and the male to female ratio was 1.2:1.

Among the patients, fifty-two had tumors (66\%) in the nasal cavity and $27(34 \%)$ at extranasal sites including the nasopharynx (ratio 2:1). Tumor biopsies were subjected to immunotyping with a panel of monoclonal antibodies against LCA, CD56, CD3, CD5, CD20, CD79 $\alpha$, CD10, CD23, Bcl-2, TIA-1, GrB, Perforin, and Ki-67. EBV-encoded RNAs were detected by in situ hybridization. All the pathologic studies were independently reviewed by three senior pathologists and a final diagnosis was made in accordance with the WHO criteria.

\subsection{Treatment and tumor responses}

After diagnosis was confirmed by pathology, patients were treated with radiotherapy and/or chemotherapy. These regimens included DHAP, IMVP-16, GMOX and $\mathrm{CHOP}-$ like regimens. The median dose of radiotherapy was $54 \mathrm{~Gy}$ (ranged $2 \mathrm{~Gy}-77 \mathrm{~Gy}$ ). 30 patients were treated with CHOP-like regimen. Drugs were given in cycles of 3 weeks and treatment lasted for 4-6 cycles.

After completion of the treatment, tumor responses were assessed according to the international standard response criteria for non-Hodgkin's lymphoma formulated in 1999. Based on the tumor imaging studies with CT scans before and after the treatment, overall responses were categorized as complete response (CR), uncertain complete response (CRu), partial response (PR), stable disease (SD), or progressive disease (PD). $A$ response rate (RR) was used to capture both $C R$ and PR.

\subsection{Long term follow-up and survival}

All patients were followed up every 3 months for the first 2 years, every 6 months for years 3-5 and once a year afterwards. At all the follow-up visits, the standard clinic evaluation for NK/T-cell lymphoma included a physical examination, a complete blood count and sedimentation ratio, blood chemistry, and CT or MRI scan (or PET/CT scan, if necessary). Sometimes nasopharyngoscope was used to differentiate relapse from fibroplasia after radiotherapy. Follow-up studies were completed for all patients in November, 2009 except for six of them who were lost to follow-up.

Overall survival (OS) was measured as the time from the start of initial treatment to patient death or last to follow-up.

\subsection{Reverse transcription}

Blood samples were drawn from all patients and healthy control subjects at the beginning of treatment. They were collected in heparin-containing tubes, and then cells were pelleted by centrifugation at $1200 \mathrm{rpm}$ for 20 minutes, and plasma was transferred into new tubes.

Reverse transcription was carried out using the plasma samples without RNA extraction according to the protocol provided by the manufacture of MMLV reverse-transcriptase reagents (Invitrogen, USA). First, a $5 \mu \mathrm{l}$ nuclease-free water solution containing $1 \mu \mathrm{l}$ of 


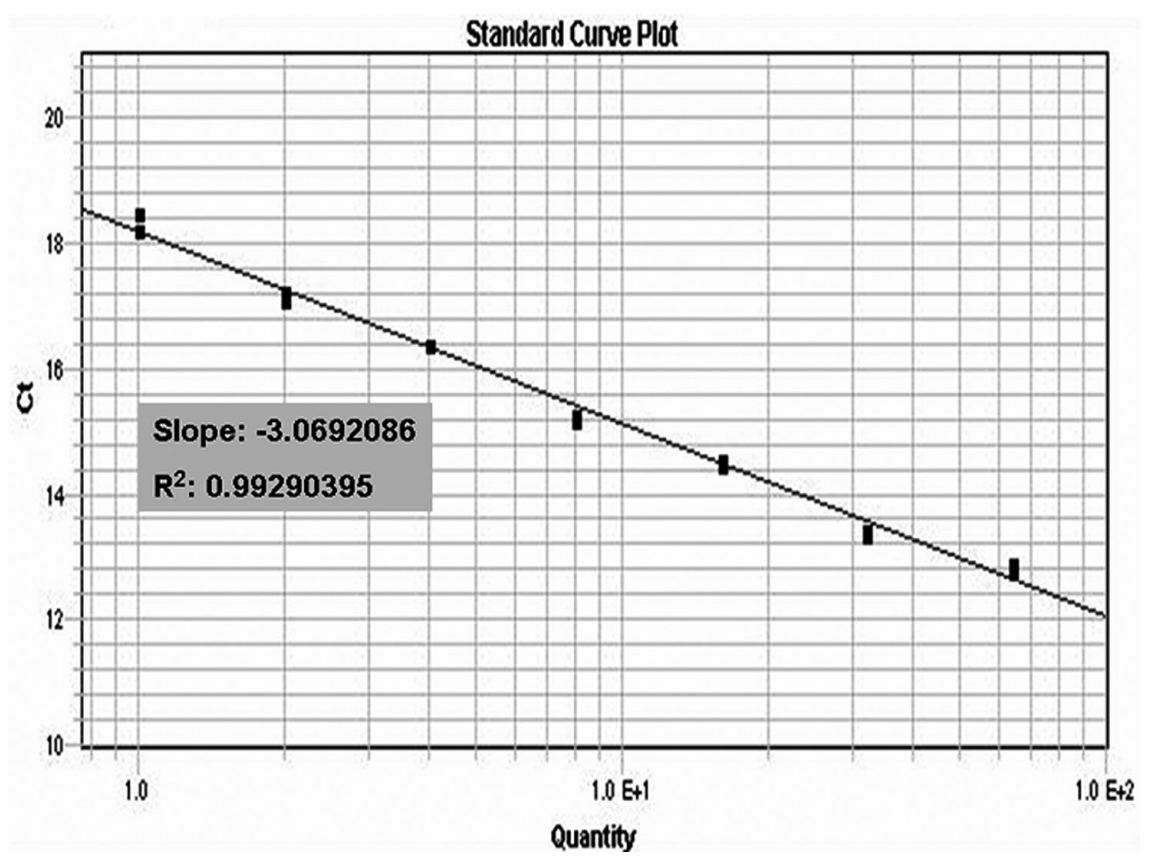

Fig. 1. Amplification curve of miR-221 by real-time PCR. The assay showed $\mathrm{C}_{T}$ values was correlated with the log of sample input over three orders of magnitude.

plasma sample, $1 \mu \mathrm{l}$ of $500 \mathrm{nM}$ stem-loop RT primer and $1 \mu \mathrm{l}$ of $10 \mathrm{mM}$ dNTPs was incubated at $65^{\circ} \mathrm{C}$ for $5 \mathrm{~min}$ and cooled on ice for $5 \mathrm{~min}$. Then, the enzyme mix containing $1 \mu \mathrm{l} 0.1 \mathrm{M}$ DTT, $2 \mu 15 \mathrm{x}$ reversetranscription buffer, $0.5 \mu 1200 \mathrm{U} / \mu 1 \mathrm{MMLV}$ reversetranscriptase, and $0.5 \mu \mathrm{l} 40 \mathrm{U} / \mu \mathrm{l}$ RNase inhibitor was added into the annealed sample. The reaction was incubated at $37^{\circ} \mathrm{C}$ for $90 \mathrm{~min}$, and then heat inactivated at $70^{\circ} \mathrm{C}$ for $15 \mathrm{~min}$. The cDNA samples were diluted to $500 \mu \mathrm{l}$ with nuclease-free water. The stem-loop RT primers for miR-221 was 5'-GTC GTA TCC AGT GCA GGG TCC GAG GTA TTC GCA CTG GAT ACG ACG AAA CC-3'.

\subsection{Quantitative real-time $P C R$}

Real-time PCR was performed using Platium SYBR Green qPCR SuperMix-UDG reagent (Invitrogen, USA) according to the manufacture's protocol and a PRISM 7900HT instrument (Applied Biosystems, US). A $15 \mu \mathrm{l}$ reaction contained $7.5 \mu \mathrm{l}$ of $2 \mathrm{xSYBR}$ Green qPCR SuperMix, $1 \mu \mathrm{l}$ each of $5 \mu \mathrm{M}$ forward and reverse primers, and $1.5 \mu \mathrm{l}$ of cDNA. The reactions were incubated in a 96 -well plate; at $95^{\circ} \mathrm{C}$ for $10 \mathrm{~min}$, followed by 45 cycles of $95^{\circ} \mathrm{C}$ for $30 \mathrm{sec}$ and $60^{\circ} \mathrm{C}$ for $1 \mathrm{~min}$. All reactions were run in duplicates. The sequences of forward and reverse primers specific for miR-221 were 5'-CGA GCT ACA TTG TCT GCT GGG T-3', 5'-CCG CAG CTA CAT CTG GCT ACT G-3' and5'GTG CAG GGT CCG AGG T-3'. $\mathrm{C}_{T}$ values were converted into relative expression levels using a standard curve which was constructed from the results of simultaneous amplifications of serial dilutions of the cDNA sample.

\subsection{Statistical analysis}

The correlation between clinicopathologic features and response rates with plasma miRNA levels was determined by Chi-square test or Student's t test. ROC curves were established in order to discriminate patients with or without NK/T-cell lymphoma. The cutoff point of plasma miRNA levels for the prediction of overall survival was determined by using the Youden index (sensitivity + specificity-1) [14]. OS was estimated using the Kaplan-Meier method. Log-rank analysis was used to compare the survival rates. Continuous biological variables were dichotomized. A prognostic model was established by fitting all the variables that significantly influenced OS at a level of $p<0.05$ in the univariate analysis. A forward stepwise Cox regression analysis was then performed. A p value less than 0.05 was considered statistically significant and all $p$ values correspond to two-sided significance tests. 

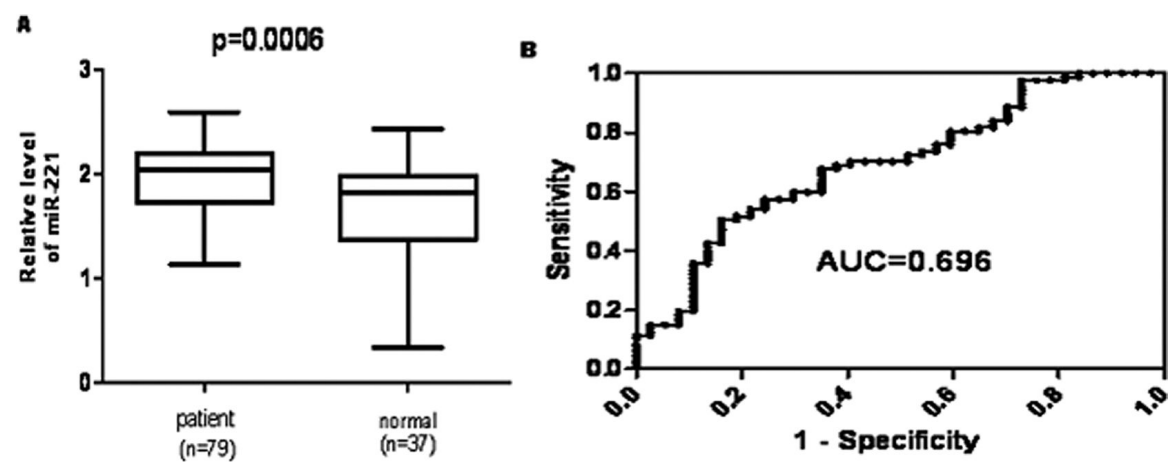

Fig. 2. Discrimination of patients with NK/T-cell lymphoma and healthy controls based on their plasma miR-221 levels. A. Plasma levels of miR-221 in healthy individuals $(n=37)$ and patients with NK/T-cell lymphoma $(n=79)$. Statistically significant differences were showed $(p=$ 0.0006). B. Receiver operating characteristics (ROC) curve analysis shows that miR-221 can discriminate patients with NK/T-cell lymphoma from healthy individuals. Plasma miR-221 yielded a ROC curve value of $0.696(95 \% \mathrm{CI}=0.556$ to 0.797$)$ with the sensitivity $57.5 \%$ and specificity of $75.7 \%$.

All above statistical analyses were performed with the SPSS software version 16.0 and Graph-pad prism 5.01 .

\section{Results}

\subsection{Level of circulating miR-221}

First, we tried to detect circulating miR-221 without RNA extraction. cDNA was directly reverse transcribed from the plasma sample, and serially diluted. Real-time PCR was performed using primers specific to miR-221 and an amplification curve was plotted with different amount of starting material from 1 to 1000 dilutions of the cDNA. A good linearity was observed between the log of sample input and $\mathrm{C}_{T}$ values in this range of dilution with the regression of 0.989 . Therefore, this PCR protocol was used for the following experiments.

Then, miR-221 levels in the plasma samples from both the patients and healthy control subjects were examined following the same real-time PCR protocol. The mean miR-221 level converted from $C_{T}$ values in the plasma from the patients was significantly higher than that from the healthy controls $(1.995 \pm 0.036 \mathrm{vs}$. $1.684 \pm 0.079, p=0.0006$, Fig. 2A). Receiver operating characteristic (ROC) curve analysis showed a ROC curve area of 0.696 , indicating that miR-221 level in the plasma can differentiate patients with NK/T-cell lymphoma from the controls $(95 \% \mathrm{CI}=0.556$ to 0.797 , Fig. 2B). At the cutoff value of 1.975 for miR-221, a sensitivity $57.5 \%$ and specificity $75.7 \%$ were obtained.

\subsection{Correlation of plasma miR-221 level with clinical features}

Plasma miR-221 levels were measured in all 79 patients before the treatment. Using the cut-off value of 1.916 which was determined by Youden index [14], 41 patients had plasma miR-221 level above and 38 cases had a level lower than the cutoff value of 1.916; therefore they were grouped into the high and low miR-221 groups, respecitvely. When comparing clinical data between these two groups, significant difference was found with respect to sex $(p=0.003)$ and ECOG Performance Status (ECOG PS; $p=0.023$ ). However, there was no significant difference in patient age ( $p=$ $0.484)$, primary sites of lesions $(p=0.152)$, levels of albumin (ALB; $p=0.312$ ) and lactate dehydrogenase (LDH; $p=0.327$ ), white blood cell counts (WBC; $p=$ 0.612 ), hematoglobin (HB; $p=0.827$ ), platelets (PLT; $p=0.179)$, statuses of Ann Arbor stage $(p=0.586)$, B symptoms $(p=0.845)$ and different treatment $(p=$ 0.074) (Table 1).

\subsection{Lack of correlation between miR-221 level and CR rate}

At the end of treatment, tumor responses to treatment were assessed for all the patients by imaging studies based on the international standard response criteria for non-Hodgkin's lymphoma. When comparing patients in the high and low miR-221 groups, essentially no significant difference was found in the response rate (RR) or the CR rate (RR: $p=0.843$; CR: $p=0.410$ ). Because up-regulation of miR-221 expression has been reported in the MCF-7/ADR cells, further analysis for 
Table 1

Clinicopathologic correlation with plasma levels of miR-221

\begin{tabular}{|c|c|c|c|}
\hline \multirow[t]{2}{*}{ Parameters } & \multicolumn{2}{|c|}{ MiR-221 expression } & \multirow[t]{2}{*}{$\mathrm{p}$} \\
\hline & Low & High & \\
\hline \multicolumn{4}{|l|}{ Sex } \\
\hline male & 14 & 40 & 0.003 \\
\hline female & 15 & 10 & \\
\hline \multicolumn{4}{|l|}{ Age } \\
\hline$>60$ & 26 & 42 & 0.484 \\
\hline$\leqslant 60$ & 3 & 8 & \\
\hline \multicolumn{4}{|c|}{ Primary sites of leisons } \\
\hline Nasal leisons & 22 & 30 & 0.152 \\
\hline Extranasal leisons & 7 & 20 & \\
\hline \multicolumn{4}{|l|}{ Ann Arbor stage } \\
\hline I & 16 & 26 & 0.586 \\
\hline II & 9 & 18 & \\
\hline III/IV & 4 & 6 & \\
\hline \multicolumn{4}{|l|}{ ECOG PS } \\
\hline $0 \sim 1$ & 29 & 42 & 0.023 \\
\hline $2 \sim 3$ & 0 & 8 & \\
\hline \multicolumn{4}{|l|}{ B symptoms } \\
\hline yes & 14 & 23 & 0.845 \\
\hline no & 15 & 27 & \\
\hline \multicolumn{4}{|l|}{ LDH } \\
\hline Normal & 19 & 38 & 0.327 \\
\hline elevated & 10 & 12 & \\
\hline \multicolumn{4}{|l|}{ ALB } \\
\hline Normal & 27 & 41 & 0.312 \\
\hline Decreased & 2 & 9 & \\
\hline \multicolumn{4}{|l|}{ WBC } \\
\hline Normal & 24 & 39 & 0.612 \\
\hline Decreased & 5 & 11 & \\
\hline \multicolumn{4}{|l|}{ HB } \\
\hline Normal & 22 & 39 & 0.827 \\
\hline Decreased & 7 & 11 & \\
\hline \multicolumn{4}{|l|}{ PLT } \\
\hline Normal & 29 & 47 & 0.179 \\
\hline Decreased & 0 & 3 & \\
\hline \multicolumn{4}{|l|}{ Different Treatment } \\
\hline CHOP-like regimens & 13 & 17 & \\
\hline other regimens & 26 & 23 & 0.074 \\
\hline
\end{tabular}

Abbreviations: ALB, albumin; CR, complete response; ECOG PS, Eastern Cooperative Oncology Group performance status; $\mathrm{Hb}$, hemoglobin; LDH, lactate dehydrogenase; PLT, platelet; WBC, white blood cell.

patients who were treated with CHOP-based regimen alone also failed to identify any significant difference between the two miR-221 groups in their RR and CR rates (RR: $p=0.088$; CR: $p=0.156$ ).

\subsection{Correlation of plasma miR-221 level with overall survival}

The prognostic value of miR-221 was investigated by comparing OS of patients between the high and low miR-221 groups. Higher plasma miR-221 level was associated with a shorter OS, an indicator of poor prognosis (Fig. 3); and the difference in the OS of the two groups was significant $(p=0.038)$. However, this difference of OS was not due to the different treatment since patients in each group received different treatment regimens (DHAP, IMVP-16, GMOX and CHOP-like regimens) assigned solely based on their clinic features that were independent on their miR-221 levels.

Univariate analysis and multivariate Cox regression were used to analyze whether plasma miR-221 and other clinical parameters could be independent prognostic factors for NK/T-cell lymphoma. First, a total of 12 parameters were analyzed with unvariate analysis. While six factors, sex $(p=0.521)$, primary sites of lesions $(p=0.450), \operatorname{ALB}(p=0.166), \mathrm{WBC}(p=0.190), \mathrm{Hb}$ $(p=0.174)$ and PLT $(p=0.263)$ at baseline showed no correlation with OS, other six factors, age ( $p=$ $0.008)$, Ann Arbor stage $(p=0.035)$, PS $(p<0.001)$, B symptoms $(p=0.001), \mathrm{LDH}(p=0.017)$ and CR after primary treatment $(p<0.001)$ display correlation with $O S$ at varying degrees.

Then, these latter six factors together with plasma miR-221 level were further analyzed with Coxregression model. Five factors, plasma miR-221 level $(p=0.003)$, age $(p<0.001)$, B symptoms $(p=0.025)$, $\mathrm{LDH}(P=0.003)$ and CR after primary treatment $(p<$ $0.001)$ were identified as independent prognostic factors for OS (Table 2).

\section{Discussion}

miRNA expression can be tissue specific, and their aberrant expression has been implicated in certain cancers. Previous studies showed that miRNA expression profile varies among tissues of different developmental origin [15], and similarly tumor samples can be sorted into different tissue origins based on their miRNA signature [16]. More recently, miR-92 level has been shown to be significantly elevated in the plasma of colorectal patients therefore it may be a molecular marker for noninvasive colorectal diagnosis [17]. By regulating tumor related genes, miRNAs function as both tumor suppressors and oncogenes in cancers. Therefore, they are referred to as oncomirs [18].

In all the earlier studies, expression data for miRNAs were generated with total RNA samples containing miRNA extracted from tissues or plasma [19-22]. The RNA extraction protocols often result in the loss of RNA and require internal controls. Therefore, we tempted to directly amplify plasma miRNAs and obtained consistent and optimal result for miR-221.

NK/T-cell lymphoma is often treated with the CHOP-based regimen but the efficacy is low because 
Table 2

Unvariate and multivariate analysis for OS

\begin{tabular}{|c|c|c|c|c|c|c|}
\hline \multirow{2}{*}{ Characteristic } & \multicolumn{3}{|c|}{ Unvariate analysis } & \multicolumn{3}{|c|}{ Multivariate analysis } \\
\hline & HR & $\mathrm{CI}(95 \%)$ & $\mathrm{p}$ value & HR & CI $(95 \%)$ & $\mathrm{p}$ value \\
\hline Plasma miR-221 levels & 0.395 & $0.165 \sim 0.950$ & 0.038 & 0.175 & $0.055 \sim 0.561$ & 0.003 \\
\hline Sex & 0.754 & $0.318 \sim 1.788$ & 0.521 & & & \\
\hline Age & 3.606 & $1.398 \sim 9.298$ & 0.008 & 9.224 & $2.709 \sim 31.409$ & $<0.001$ \\
\hline ECOG PS & 6.064 & $2.332 \sim 15.765$ & $<0.001$ & 2.001 & $0.601 \sim 6.665$ & 0.258 \\
\hline B symptoms & 11.058 & $1 \sim 0.001$ & 0.001 & 3.364 & $1.168 \sim 9.688$ & 0.025 \\
\hline $\mathrm{Hb}$ & 1.838 & $0.764 \sim 4.410$ & 0.174 & & & \\
\hline WBC & 1.739 & $0.760 \sim 3.979$ & 0.190 & & & \\
\hline PLT & 3.200 & $0.417 \sim 24.575$ & 0.263 & & & \\
\hline LDH & 2.551 & $1.182 \sim 5.509$ & 0.017 & 4.933 & $1.732 \sim 14.049$ & 0.003 \\
\hline ALB & 2.138 & $0.729 \sim 6.271$ & 0.166 & & & \\
\hline Ann Arbor stage & 1.462 & $1.027 \sim 2.080$ & 0.035 & 1.192 & $0.816 \sim 1.741$ & 0.363 \\
\hline Primary sites & 1.371 & $0.604 \sim 3.111$ & 0.450 & & & \\
\hline $\mathrm{CR}$ after primary therapy & 4.868 & $2.128 \sim 11.133$ & $<0.001$ & 9.681 & $3.120 \sim 30.036$ & $<0.001$ \\
\hline
\end{tabular}

Abbreviations: HR, Hazard Ratio; CI, Confidence interval. Statistical significance: $p<0.05$.

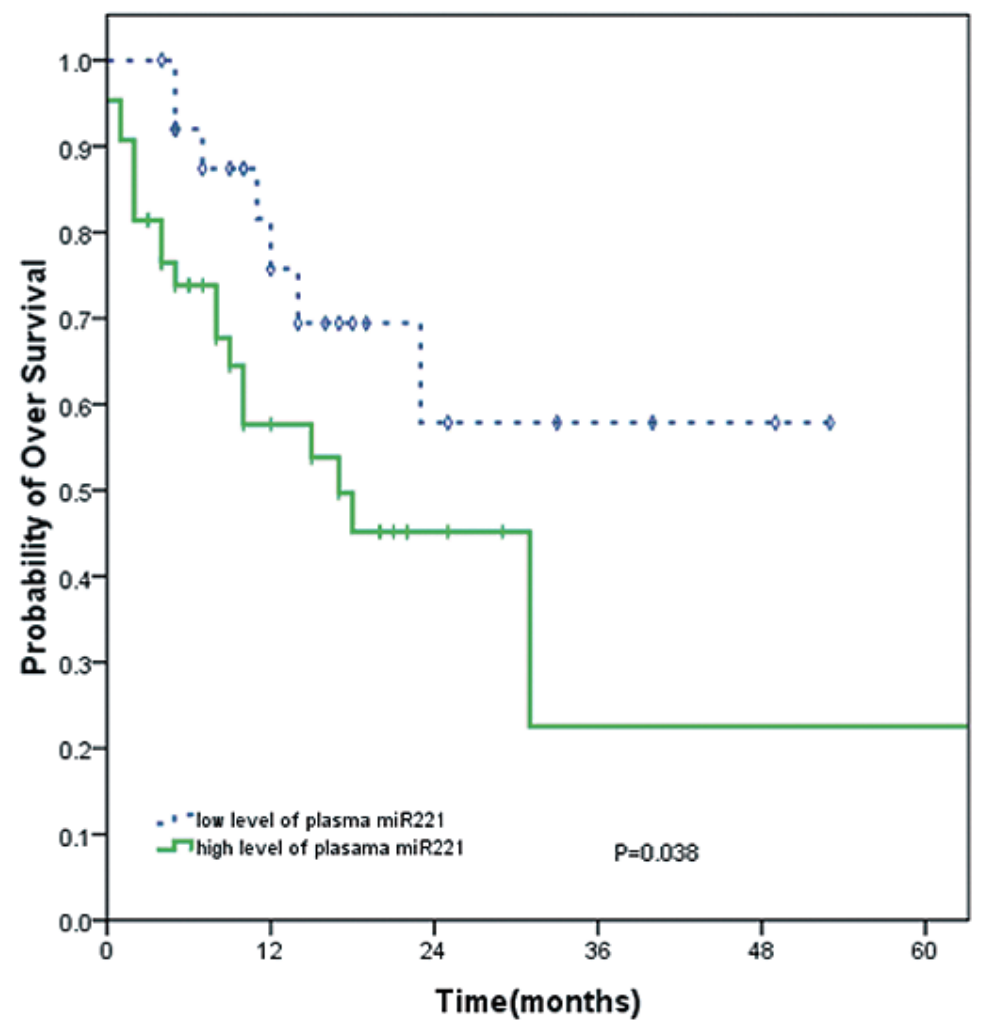

Fig. 3. Correlation of Overall Survival rate for patients with NK/T-cell lymphoma with their plasma miR-221 level. A significantly lower survival rate was observed in patients in the high plasma miR-221 group than in the low miR-221 group $(p<0.05)$.

of high expression of P-gp rendering the resistance of tumor to adriamycin, a substrate of p-gp [23]. Because elevated expression of miRNA-221 has been shown in cell lines resistant to adriamycin, we selected it for the study aiming to evaluate their potential values in diagnosis and prognosis of NK/T-cell lymphoma. By screening samples from 79 patients with NK/T-cell lymphoma and 37 healthy subjects, a significantly higher plasma miR-221 level was identified in patients than in the healthy individuals. 
Park JK et al. [24] reported that antisense oligonucleotides (ASO) against miR-221 can enhance chemosensitivity of gemcitabine by up-regulating p 27 expression in human pancreatic cancer cell lines. This suggests a potential contribution of miR-221 to the poor response to chemotherapy. Since most NK/T-cell lymphomas are negative for p27 [25,26], and the expression of p27 is also low in the NK/T-cell lymphoma cell line (Hank1); therefore, we compared response and CR rates between patients in the low and high miR-221 groups. However, no correlation was found between plasma miRNA-221 level and RR or CR rate. This could be due to the relatively small number of patients and varied treatment modality. When patients treated with CHOP-like regimen alone were analyzed, although the difference in the RR or CR between two groups was still below the significant level, the $\mathrm{p}$ value was close to 0.05 . Therefore, analysis with a larger sample size may probably generate a significant difference.

In human melanoma cell lines, overexpression of miR-221 has been shown to increase cell proliferation, anchorage-independent growth, invasion and migration and reduce cellular differentiation [27]. Overexpression of miR-221 also enhances proliferation and tumorigenicity of poorly aggressive LNCaP cells, and inhibition of miR-221 significantly slows down tumor growth in a mouse model [28]. Since proliferation of malignant cells contribute to the tumors progression in human, and can ultimately affect performance status (PS) and survival of the patients, our observation that a higher plasma miR-221 level is associated with a poorer performance is consistent with its tumor-promoting roles. In addition, the correlation of a high miR-221 with a poor OS indicates that miR-221 can be a prognostic factor for NK/T-cell lymphoma.

In conclusion, our analysis showed that level of miR221 is elevated in the circulation of NK/T-cell lymphoma patients, and a higher plasma miR-221 level is associated with a poorer performance at baseline and a less favorable long term outcome. Based on these results, plasma miR-221 may serve as a diagnostic and prognostic marker, and be used one day as a therapeutic target for the treatment of NK/T-cell lymphoma.

\section{Acknowledgments}

This study was supported by grants from the Nature Science foundation of Guangdong Province (05200178), the Research Fund for the Dectoral
Program of Higher Education (200805580080) and Projects under Scientific and Technological Planning of Guangzhou City (2006Z3-E0021), China.

\section{References}

[1] T.M. Kim, Y.H. Park, S.Y. Lee, J.H. Kim, D.W. Kim, S.A. Im, T.Y. Kim, C.W. Kim, D.S. Heo, Y.J. Bang, K.H. Chang and N.K. Kim, Local tumor invasiveness is more predictive of survival than International Prognostic Index in stage $\mathrm{I}(\mathrm{E}) / \mathrm{II}(\mathrm{E})$ extranodal NK/T-cell lymphoma, nasal type, Blood 106 (2005), 3785-3790.

[2] W. Yong, W. Zheng, Y. Zhang, J. Zhu, Y. Wei, D. Zhu and J. Li, $\mathrm{L}$-asparaginase-based regimen in the treatment of refractory midline nasal/nasal-type T/NK-cell lymphoma, Int J Hematol 78 (2003), 163-167.

[3] K. Isobe, T. Uno, J. Tamaru, H. Kawakami, N. Ueno, H. Wakita, J. Okada, J. Itami and H. Ito, Extranodal natural killer/T-cell lymphoma, nasal type: the significance of radiotherapeutic parameters, Cancer 106 (2006), 609-615.

[4] X.X. Zhang, C.H. Xie, Y. Xu, D. Deng, Y.H. Zhao, B.W. Zou, L. Zhou, M. Li, J. Wang, W.P. Liu and M.J. Huang, Salvage Treatment Improved Survival of Patients with Relapsed Extranodal Natural Killer/T-Cell Lymphoma, Nasal Type, Int J Radiat Oncol Biol Phys 74 (2009), 747-752.

[5] I. Behm-Ansmant, J. Rehwinkel and E. Izaurralde, MicroRNAs silence gene expression by repressing protein expression and/or by promoting mRNA decay, Cold Spring Harb Symp Quant Biol 71 (2006), 523-530.

[6] D.P. Bartel, MicroRNAs: genomics, biogenesis, mechanism, and function, Cell 116 (2004), 281-297.

[7] R.C. Lee, R.L. Feinbaum, V. Ambros and C. The, elegans heterochronic gene lin-4 encodes small RNAs with antisense complementarity to lin-14, Cell 75 (1993), 843-854.

[8] B. Wightman, I. Ha and G. Ruvkun, Posttranscriptional regulation of the heterochronic gene lin-14 by lin- 4 mediates temporal pattern formation in C. elegans, Cell 75 (1993), 855862.

[9] W.P. Kloosterman and R.H. Plasterk, The diverse functions of microRNAs in animal development and disease, Dev Cell 11 (2006), 441-450.

[10] P. Carninci, T. Kasukawa, S. Katayama, J. Gough, M.C. Frith, N. Maeda, R. Oyama, T. Ravasi, B. Lenhard, C. Wells and R. Kodzius, The transcriptional landscape of the mammalian genome, Science 309 (2005), 1559-1563.

[11] C.M. Croce and G.A. Calin, miRNAs, cancer, and stem cell division, Cell 122 (2005), 6-7.

[12] X.Y. Cui, Y.J. Guo and H.R. Yao, Analysis of microRNA in drug-resistant breast cancer cell line MCF-7/ADR, J South Med Univ, China 28 (2008), 1813-1815.

[13] O. Kovalchuk, J. Filkowski, J. Meservy, Y. Ilnytskyy, V.P. Tryndyak, V.F. Chekhun and I.P. Pogribny, Involvement of microRNA-451 in resistance of the MCF-7 breast cancer cells to chemotherapeutic drug doxorubicin, Mol Cancer Ther 7 (2008), 2152-2159.

[14] W.J. Youden, Index for rating diagnostic tests, Cancer 3 (1950), 32-35.

[15] J. Lu, G. Getz, E.A. Miska, E. Alvarez-Saavedra, J. Lamb, D. Peck, A. Sweet-Cordero, B.L. Ebert, R.H. Mak, A.A. Ferrando, J.R. Downing, T. Jacks, H.R. Horvitz and T.R. Golub, MicroRNA expression profiles classify human cancers, Nature 435 (2005), 834-848. 
[16] S. Volinia, G.A. Calin, C.G. Liu, S. Ambs, A. Cimmino, F Petrocca, R. Visone, M. Iorio, C. Roldo, M. Ferracin, R.L. Prueitt, N. Yanaihara, G. Lanza, A. Scarpa, A. Vecchione, M. Negrini, C.C. Harris and C.M. Croce, A microRNA expression signature of human solid tumors defines cancer gene targets, Proc Natl Acad Sci USA 103 (2006), 2257-2261.

[17] E.K. Ng, W.W. Chong, H. Jin, E.K. Lam, V.Y. Shin, J. Yu, T.C. Poon, S.S. Ng and J.J. Sung, Differential expression of microRNAs in plasma of patients with colorectal cancer: a potential marker for colorectal cancer screening, Gut 58 (2009), 1375-1381.

[18] A. Esquela-Kerscher and F.J. Slack, Oncomirs - microRNAs with a role in cancer, Nat Rev Cancer 6 (2006), 259-269.

[19] S.S. Chim, T.K. Shing, E.C. Hung, T.Y. Leung, T.K. Lau, R.W. Chiu and Y.M. Lo, Detection and Characterization of Placental MicroRNAs in Maternal Plasma, Clin Chem 54 (2008), 482490.

[20] P.S. Mitchell, R.K. Parkin, E.M. Kroh, B.R. Fritz, S.K. Wyman, E.L. Pogosova-Agadjanyan, A. Peterson, J. Noteboom, K.C. O'Briant, A. Allen, D.W. Lin, N. Urban, C.W. Drescher, B.S. Knudsen, D.L. Stirewalt, R. Gentleman, R.L. Vessella, P.S. Nelson, D.B. Martin and M. Tewari, Circulating microRNAs as stable blood-based markers for cancer detection, Proceedings of the National Academy of Sciences $\mathbf{1 0 5}$ (2008), 10513-10518.

[21] C.H. Lawrie, S. Gal, H.M. Dunlop, B. Pushkaran, A.P. Liggins, K. Pulford, A.H. Banham, F. Pezzella, J. Boultwood, J.S. Wainscoat, C.S. Hatton and A.L. Harris, Detection of elevated levels of tumour-associated microRNAs in serum of patients with diffuse large B-cell lymphoma, Br J Haematol 141 (2008), 672-675.

[22] X. Chen, Y. Ba, L. Ma, X. Cai, Y. Yin, K. Wang, J. Guo, Y. Zhang, J. Chen, X. Guo, Q. Li, X. Li, W. Wang, Y. Zhang,
J. Wang, X. Jiang, Y. Xiang, C. Xu, P. Zheng, J. Zhang, R. Li, H. Zhang, X. Shang, T. Gong, G. Ning, J. Wang, K. Zen, J. Zhang and C.Y. Zhang, Characterization of microRNAs in serum: a novel class of biomarkers for diagnosis of cancer and other diseases, Cell Res 18 (2008), 997-1006.

[23] B. Wang, X.Q. Li, X. Ma, X. Hong, H. Lu and Y. Guo, Immunohistochemical expression and clinical significance of P-glycoprotein in previously untreated extranodal NK/T-cell lymphoma, nasal type, Am J Hematol 83 (2008), 795-799.

[24] J.K. Park, E.J. Lee, C. Esau and T.D. Schmittgen, Antisense Inhibition of microRNA-21 or -221 Arrests Cell Cycle, Induces Apoptosis, and Sensitizes the Effects of Gemcitabine in Pancreatic Adenocarcinoma, Pancreas 38 (2009), 191-199.

[25] M. Xiang-Lan, S. Zu-Lan, H. Dan, S. Bi-Hong, P. Ya-Qin and L. Han-Liang, Skp2/p27 expression profile is correlated with Epstein-Barr virus status in extranodal nasal-type natural killer cell lymphoma, Transl Res 151 (2008), 303-308.

[26] M.X. Ian, S.Z. Lan, Z.F. Cheng, H. Dan and L.H. Qiong, Suppression of EBNA1 expression inhibits growth of EBVpositive NK/T cell lymphoma cells, Cancer Biol Ther 7 (2008), 1602-1606.

[27] F. Felicetti, M.C. Errico, L. Bottero, P. Segnalini, A. Stoppacciaro, M. Biffoni, N. Felli, G. Mattia, M. Petrini, M.P. Colombo, C. Peschle and A. Car, The promyelocytic leukemia zinc finger-microRNA-221/-222 pathway controls melanoma progression through multiple oncogenic mechanisms, Cancer Res 68 (2008), 2745-2754.

[28] N. Mercatelli, V. Coppola, D. Bonci, F. Miele, A. Costantini, M. Guadagnoli, E. Bonanno, G. Muto, G.V. Frajese, R. De Maria, L.G. Spagnoli, M.G. Farace and S.A. Ciafr, The inhibition of the highly expressed miR-221 and miR-222 impairs the growth of prostate carcinoma xenografts in mice, PLoS One 3 (2008), e4029. 


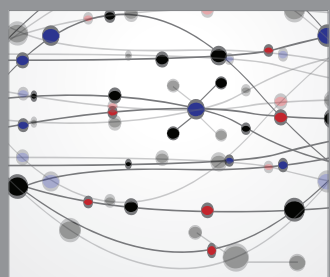

The Scientific World Journal
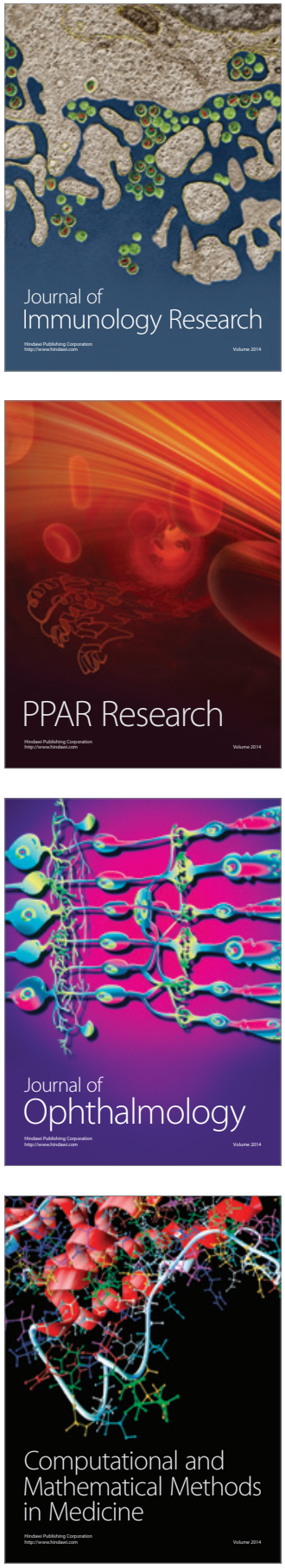

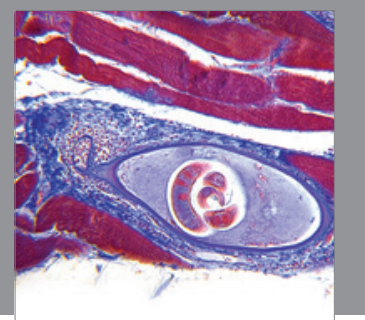

Gastroenterology

Research and Practice
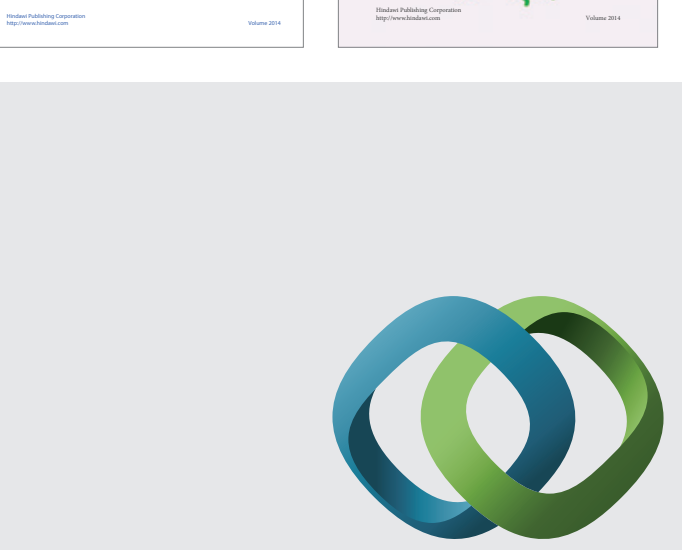

\section{Hindawi}

Submit your manuscripts at

http://www.hindawi.com
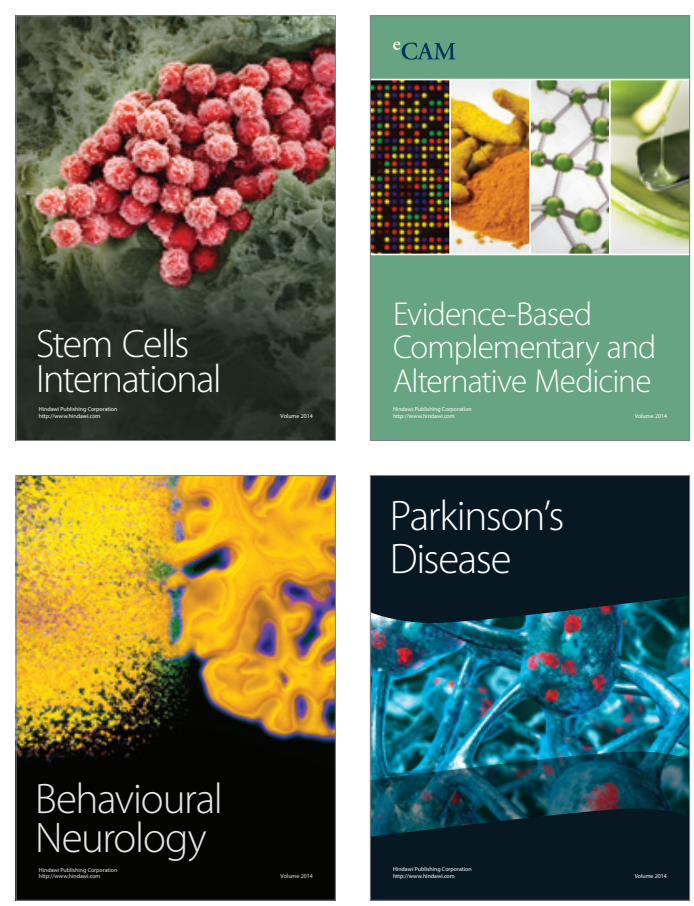

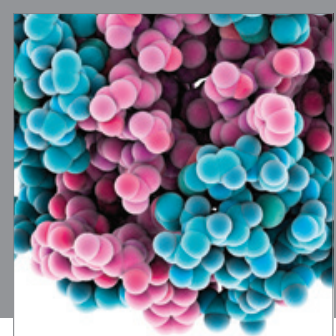

Journal of
Diabetes Research

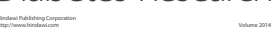

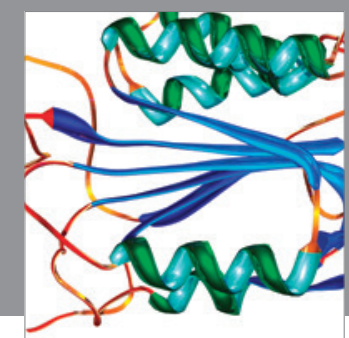

Disease Markers
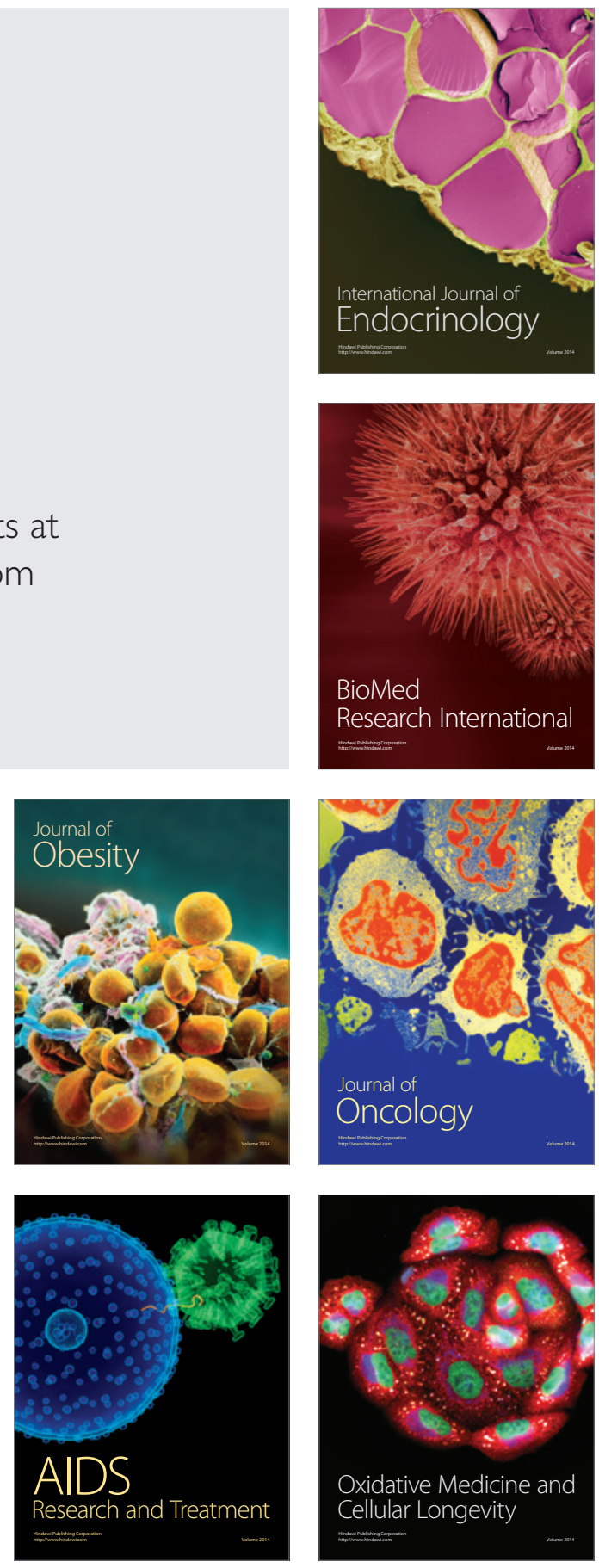